\title{
Apropiación del proceso de innovación en comunidades rurales femeninas: Caso Sotaquirá
}

\author{
Appropriation of the innovation process in rural \\ women's communities: Sotaquirá case
}

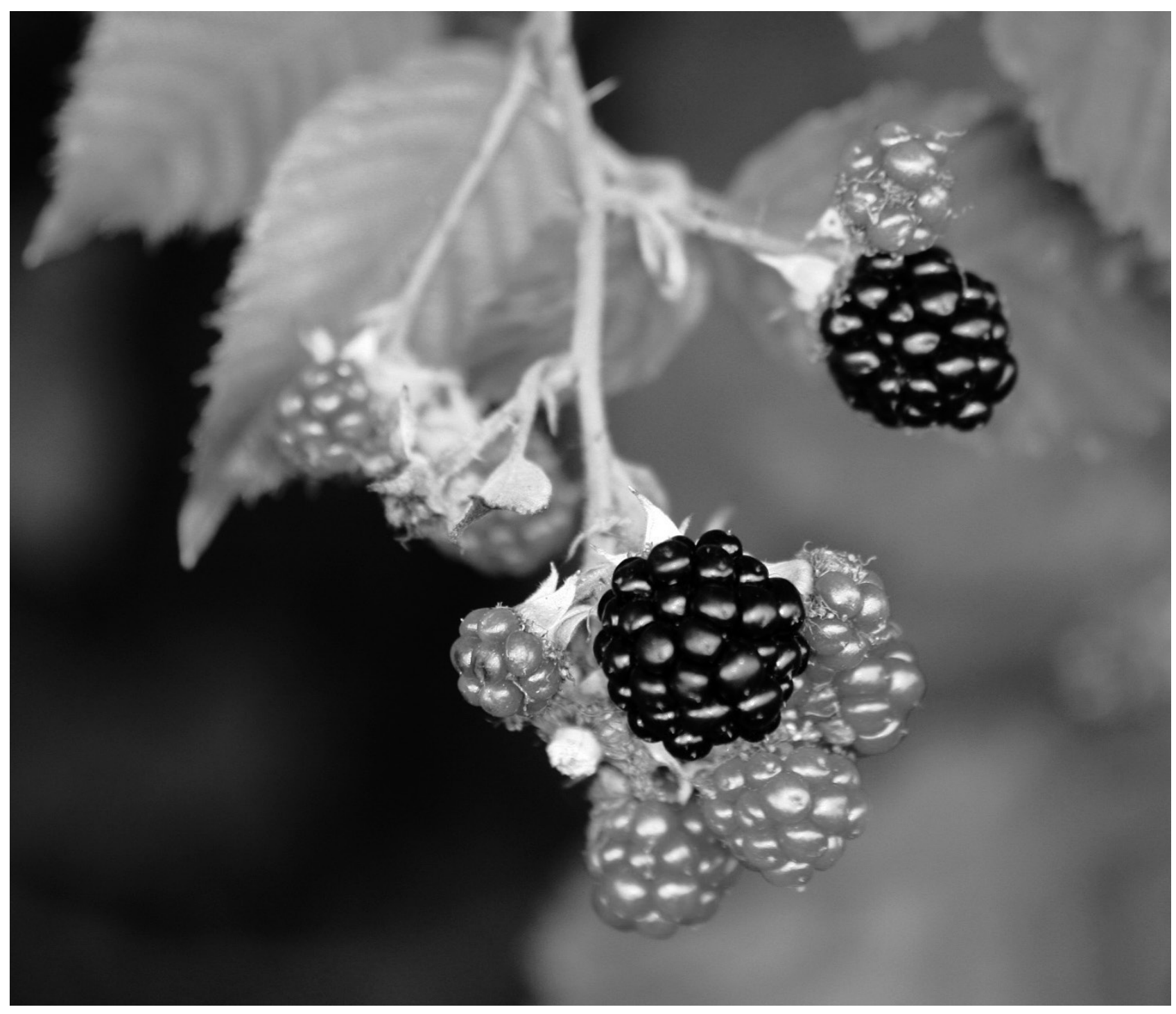




\title{
Apropiación del proceso de innovación en comunidades rurales femeninas: Caso Sotaquirá ${ }^{1}$ Appropriation of the innovation process in rural women's communities: Sotaquirá case
}

\author{
Andres Ferney Largo-León², Edwin Yesid Gómez-Pachón ${ }^{3}$
}

Artículo recibido en marzo 28 de 2019; artículo aceptado en mayo 08 de 2019.

Este artículo puede compartirse bajo la Licencia Creative Commons Atribución-NoComercial-Compartirlgual 4.0 Internacional y se referencia usando el siguiente formato: Largo-León, A. F. \& Gomez-Pachon, E. Y. (2019). Apropiación del proceso de innovación en comunidades rurales femeninas: Caso Sotaquirá. I+D Revista de Investigaciones, 14 (2), 87-101.

DOI: https://doi.org/10.33304/revinv.v14n2-2019008

\section{Resumen}

El presente artículo presenta el estudio de caso desarrollado con la Asociación Agropecuaria de Mujeres Emprendedoras del Municipio de Sotaquirá (ASOAMMES), en el que se propuso y se desarrolló una estrategia de diseño participativo, con el objetivo de generar valor agregado y ventajas competitivas en las actividades productivas que desempeñan las 40 mujeres que conforman dicha asociación. Para dar cumplimiento a este objetivo, la fase inicial correspondió a la caracterización de la comunidad e identificación de problemáticas susceptibles de solucionar, a través de un proceso de diseño participativo. Seguido de esto, se implementó una metodología ágil de codiseño llamada Design Sprint. El resultado más relevante de esta investigación fue la apropiación de la metodología de innovación por parte de la comunidad objeto de estudio. Esta apropiación se evidenció mediante el desarrollo de un dispositivo cosechador de mora, una línea de empaques para productos orgánicos y un método para elaborar dispositivos caseros para el control del caracol.

Palabras clave: Diseño participativo, innovación, mujeres rurales, empoderamiento, agricultura.

\begin{abstract}
This article presents the case study developed with the Agricultural Association of Women Entrepreneurs of the Municipality of Sotaquirá (ASOAMMES), in which a participatory design strategy was proposed and developed with the aim of generating added value and competitive advantages in the productive activities performed by 40 women that make up this association. In order to fulfill this objective, the initial phase corresponded to the

1. Artículo de estudio de caso, enfoque cualitativo, resultado de un proyecto de investigación finalizado, perteneciente al área de diseño, desarrollado en el grupo de investigación DITMAV y financiado por el Instituto de Gestión, Innovación y Aceleración de Negocios (GINNOA), de la ciudad de Tunja (Colombia). Dirección: UPTC, Duitama, carrera 18 n. 22, PBX: (8) 7 7604100. Fecha de inicio: enero de 2018. Fecha de terminación: febrero de 2019.

2. Magíster en Diseño, Universidad Pedagógica y Tecnológica de Colombia. Vinculado al Grupo de Investigación Diseño, Innovación y Asistencia Técnica de materiales Avanzados (DITMAV), de la Universidad Pedagógica y Tecnológica de Colombia, sede Duitama (Colombia). Dirección: UPTC, Duitama, carrera 18 n. 22, PBX:0(8) 7 7604100. ORCID ID: http://orcid.org/0000-0002-3065-0261. Correo electrónico: andresferney@hotmail.com.

3. Ph.D. Ciencia e Ingenieria de Materiales, Universidad Nacional Autónoma de México. Docente de la Escuela de Diseño Industrial. Vinculado al Grupo de Investigación Diseño, Innovación y Asistencia Técnica de materiales Avanzados (DITMAV), Universidad Pedagógica y Tecnológica de Colombia, sede Duitama (Colombia). Dirección: UPTC, Duitama, carrera 18 n. ${ }^{\circ}$ 22, PBX:0383107868975. ORCID ID: http://orcid.org/0000-0002-27335252. Correo electrónico institucional: edwin.gomez02@uptc.edu.co.
\end{abstract}


characterization of the community and the identification of problems that could be solved through a participatory design process. After this, an agile co-design methodology called Design Sprint was implemented. The most relevant result of this research was the appropriation of the methodology of innovation by the community under study. This appropriation was evidenced by the development of a blackberry harvester device, a line of packaging for organic products, and a method to develop homemade devices for the snail control.

Keywords: Participatory design, innovation, rural women, empowerment, farming.

\section{Introducción}

Diversos estudios y estadísticas en los ámbitos latinoamericano y mundial (Castillo, 2011) indican que los ámbitos de desenvolvimiento del trabajo y negocios de las mujeres corresponden a esferas tradicionales de producción y comercialización. Esferas que están marcadas por la división del trabajo por género, en la que hombres y mujeres cumplen tareas y roles diferenciados. Estos estudios también afirman que infortunadamente los productos de las mujeres rurales son básicos y cuentan con poco valor agregado, a pesar de que los Objetivos de Desarrollo Sostenibles (ODS) de la ONU han propuesto estrategias para disminuir las brechas entre el sector urbano y rural, así como la desigualdad de género entre hombres y mujeres (Naciones Unidas en Colombia, 2018). En anteriores políticas públicas de Colombia, como el Plan de Desarrollo 2010-2014, se priorizó al sector agropecuario como una de las locomotoras económicas que más aportan al producto interno bruto (PIB) (Olarte, 2015).

Diagnósticos en Latinoamérica y en Colombia que han medido el grado de vulnerabilidad que presentan estas comunidades rurales han evidenciado bajos índices de educación, productividad, calidad de vida y de asistencia médica (CORPOICA, 2015). En Colombia, las mujeres localizadas en las áreas rurales encuentran amplias dificultades para explotar sus capacidades, lo que frena el desarrollo integral de los territorios. Sin embargo, muchas veces esta realidad queda escondida, y esto perpetúa el retraso del desarrollo social (Botello-Peñalosa \& Guerrero-Rincon, 2017). Los ODS no han llegado a incidir sustancialmente en el desarrollo productivo y calidad de vida de las mujeres rurales, ya que las mujeres se enfrentan a la división del trabajo remunerado y no remunerado -por ende, las afecta el uso diferenciado del tiempo-, en el que la mayoría de las mujeres emplean gran parte del tiempo en servicios de cuidado de otros miembros familiares. Cuando las mujeres rurales desempeñan trabajos remunerados, sus salarios suelen ser diferenciados en relación con los de los hombres, o son trabajos mal pagados -como aquellos que se realizan en el hogar-. Una causa de esta realidad es la falta de oportunidades que tienen las mujeres de acceder a servicios de salud, actividades laborales y educación.
Específicamente la comunidad objeto de estudio es la Asociación Agropecuaria de Mujeres Emprendedoras del Municipio de Sotaquirá (ASOAMMES). Esta asociación está conformada por 40 mujeres que desempeñan actividades productivas en pequeña escala relacionadas con la agricultura. ASOAMMES es una asociación con 3 años de constitución, que aparece con el objetivo de fortalecer las actividades productivas de mujeres rurales, a través de visibilización en el mercado y capacitación en procesos productivos.

Dicha asociación ha participado en diferentes programas de capacitación, tales como buenas prácticas agrícolas, producción orgánica de frutas y verduras, entre otros. A su vez, ha participado en capacitaciones que son ofrecidas por entidades gubernamentales y particulares, como ASOHOFRUCOL. El objetivo de los programas de capacitación ha sido transferir una información que posteriormente sería implementada en los procesos productivos, ya sea mediante el cambio de procedimientos o mediante la tecnificación, con la adquisición de equipo y maquinaria. En gran parte de estos procesos, se hace un diagnóstico de los problemas productivos y se sugieren posibles soluciones; sin embargo, la asociación ha manifestado que las soluciones han sido difíciles de implementar, ya que fueron concebidas para utilizarse en otros contextos, y no se cuenta con los recursos económicos necesarios.

En este sentido, las políticas públicas siguen asumiendo el desarrollo rural como un proceso por el que las sociedades rurales se adaptan a los requerimientos de una sociedad predominantemente urbana. Las soluciones que se proponen se basan en la especialización productiva, la racionalización de los procesos, las economías de escala y la mercantilización de los intercambios, a través de la agroindustria, la minería o el turismo rural. Esto ocurre especialmente en el caso latinoamericano, en el que se solapan los objetivos de superación de la pobreza rural con los de desarrollo nacional (Romera, Suarez \& Hernandez, 2017).

Por lo anterior, esta investigación buscó plantear e implementar una estrategia de diseño con enfoque participativo que impulsara en la comunidad la capacidad de generar valor agregado y ventajas 
competitivas (Muñoz, Peña \& Vargas, 2017) en los productos y procesos productivos que desempeñan quienes conforman ASOAMMES. Para dar cumplimiento a este objetivo general, se establecieron los siguientes objetivos específicos:

I. Identificar el contexto social y económico de las mujeres rurales de Sotaquirá.

II. Identificar y priorizar las actividades productivas susceptibles de mejoramiento de valor agregado que desarrollan las mujeres rurales de Sotaquirá.

III. Desarrollar estrategias de diseño que permitan la participación de la comunidad ASOAMMES en la generación de innovación de productos y procesos que sustentan los ingresos económicos de las mujeres rurales.

IV. Evaluar la participación de la comunidad ASOAMMES y el aporte a la innovación, bajo la estrategia de diseño participativo.

Teniendo en cuenta el rol social que desempeñan las instituciones educativas, se hace necesario atender las necesidades de la sociedad y especialmente de las comunidades rurales (Paz, Sanchez \& Sanchez, 2018). Más aún si se parte del diseño, disciplina en la cual el factor social ha estado históricamente presente, como se demuestra en las diversas investigaciones realizadas por los académicos John Ruskin, William Morris y Víctor Papanek. Sin embargo, solo en años recientes se ha explorado la estimulación de comportamientos que permitan afrontar nuevos retos, como los sociales (Nicolás, 2016).

En las últimas décadas, la investigación en diseño ha identificado y ha sugerido enfoques, como el diseño centrado en la persona y el diseño para la innovación social. Estos dos enfoques se centran en el cambio social, a través de un proceso de codiseño, y el rol social puede ser el fin central de una solución. Se pasa de un factor periférico a uno estructural, y esto se traduce en bienestar común.

Las necesidades de las personas han sido frecuentemente desatendidas por los diseñadores. Una alternativa para que dicha satisfacción no quede solo en el discurso es el diseño para la innovación social. Esta se entiende como la transformación creativa de las relaciones sociales hacia nuevos modos de intercambio de valor entre personas y organizaciones (Escuela de diseño social, 2018).

Los diseñadores contribuyen actualmente con la implementación de la innovación social, ya que tienen la capacidad de crear soluciones balanceadas entre lo tecnológicamente posible y lo socialmente deseable. Manzini (2015) sugiere cuatro roles que el diseñador desempeña en un proceso de innovación social: facilitador de la innovación social, a través de procesos de codiseño, activista, estratega y promotor cultural.

La innovación social no es necesariamente la creación de una idea completamente nueva; se trata más bien de una reorganización de los elementos existentes para obtener un mejor provecho de ellos, con el fin de mejorar áreas de la vida cotidiana. Un punto de coincidencia de la literatura especializada en innovación social es que las personas son seres activos y, por tanto, partícipes potenciales en la solución de los retos planteados. Los diseñadores pueden estimular las condiciones para que esto se produzca (Manzini, 2015).

Este estudio de caso se enmarca en un proceso de investigación-acción participativa. De acuerdo con la estrategia de diseño participativo planteada, este proceso sugiere que el investigador tome el rol de facilitador entre la comunidad y la metodología. Esta investigación se hace pertinente, ya que hoy en día se requieren nuevas estrategias para hacer de las comunidades productivas organizaciones sociales más competitivas. Una de ellas es el conocimiento o capital intelectual. Es decir, que las organizaciones deben hacer uso de sus conocimientos para incrementar su competitividad (Muñoz, Peña \& Vargas, 2017).

\section{Metodología}

\section{Tipo de estudio}

El método abordado para el desarrollo de esta investigación fue el de investigación-acción participativa (IAP), ya que es un método que combina el proceso de conocer y el de actuar. Durante el proceso de investigación, fue indispensable el trabajo participativo de la comunidad, tanto para analizar la realidad en que se encuentra inmersa como para la construcción de soluciones a las problemáticas o necesidades identificadas. Este método de investigación genera un empoderamiento de la población implicada, ya que hace evidentes las capacidades, conocimientos y fortalezas que puede aportar cada individuo a la construcción de soluciones (Colmenares, 2012).

Este método de investigación ha sido tomado por diferentes iniciativas que están comprometidas con la lucha contra la pobreza y la desigualdad social. El método fue tomado de Paulo Freire (1970); de manera específica, 
de su obra titulada Pedagogía del oprimido, en la que argumenta que, por medio de la IAP, es posible consolidar y resaltar conocimientos y capacidades de los individuos. Esta perspectiva contribuyó a fortalecer el trabajo con sectores sociales vulnerables y desarrollar el concepto de 'educación popular' (Eizaguirre \& Zabala, 2019).

En este caso de estudio se contemplaron tres fases:

1. Investigación participativa

2. Acción participativa

3. Evaluación

Esta investigación es de tipo descriptiva, ya que buscó caracterizar una comunidad y presentar un plan de trabajo mediante el cual se contribuyera al desarrollo de soluciones de innovación.

\section{Participantes}

La Asociación Agropecuaria de Mujeres Emprendedoras del Municipio de Sotaquirá (ASOAMMES), en el año 2018, cuenta con 40 socias activas. Esta organización fue seleccionada para este estudio, ya que está conformada por mujeres que viven y trabajan en el municipio de Sotaquirá, y sus actividades productivas se enfocan en el sector agropecuario.

Facilitador: Es el investigador principal en este caso de estudio, quien, a su vez, es diseñador industrial y cursó estudios de posgrado en la Maestría en Diseño con énfasis en proyectos sociales. Esta persona también ha sido formada como gestor de innovación en el ecosistema de innovación de Medellín.

Entre las actividades del facilitador están:

- Planificar el proceso de investigación.

- Aplicar herramientas de recolección de información.

- Gestionar espacios requeridos para el desarrollo de los talleres participativos que fueron proporcionados por la Alcaldía del municipio de Sotaquirá.

- Coordinar y planificar las actividades a desarrollar con la comunidad.

- Gestionar los equipos tecnológicos requeridos; es decir, computadores con acceso a internet y proyectores de imagen.

- Liderar los talleres con la comunidad, mediante la transferencia de conceptos y el desarrollo de actividades. - Evaluar los resultados del proceso.

Colaboradores: Son dos personas que apoyan los talleres participativos. Para este caso, no tuvieron una formación profesional específica, pero sí fue necesario dar a conocer la metodología antes de cada sesión de trabajo. Entre sus funciones estaban:

- Ocuparse de la logística del desarrollo de cada sesión de trabajo con la comunidad.

- Apoyar al facilitador con la resolución de dudas que presente la comunidad.

- Colaborar con los grupos de trabajo en el diligenciamiento de formatos y búsquedas de información.

- Diligenciar diarios de campo en cada sesión de trabajo.

- Retroalimentar al facilitador con los pormenores de cada sesión de trabajo.

\section{Materiales e instrumentos}

Los siguientes instrumentos de investigación se utilizaron para dar cumplimiento a los objetivos de este caso de estudio:

\section{Encuesta}

Este instrumento se utilizó para dar cumplimiento al objetivo específico 1, que consiste en identificar el contexto socioeconómico que caracteriza a la población objeto de estudio. Esta herramienta se diseñó con un total de 20 preguntas, y se aplicó en una asamblea general de socias.

\section{Observación participante}

La observación participante está definida como el proceso para establecer relación con una comunidad y aprender a actuar al punto de mezclarse en ella, de forma que sus miembros actúen de forma natural (Kawulich, 2006).

Esta técnica sirvió para dar seguimiento al nivel de participación de cada una de las personas en los talleres de diseño participativo, con el objetivo de incentivar la participación de todos los integrantes por grupo de trabajo.

\section{Procedimiento}

Para dar cumplimiento a los objetivos, el primer paso a desarrollar fue convocar a las mujeres que conforman ASOAMMES, para que participaran en el desarrollo de este proyecto. Además, para dar cumplimiento al primer objetivo, se recolectó información, a partir de planes de desarrollo locales y nacionales relacionados con la proyección del municipio de Sotaquirá. Se indagó con autoridades municipales, y se aplicó una encuesta para conocer variables específicas de la población objeto de estudio. 


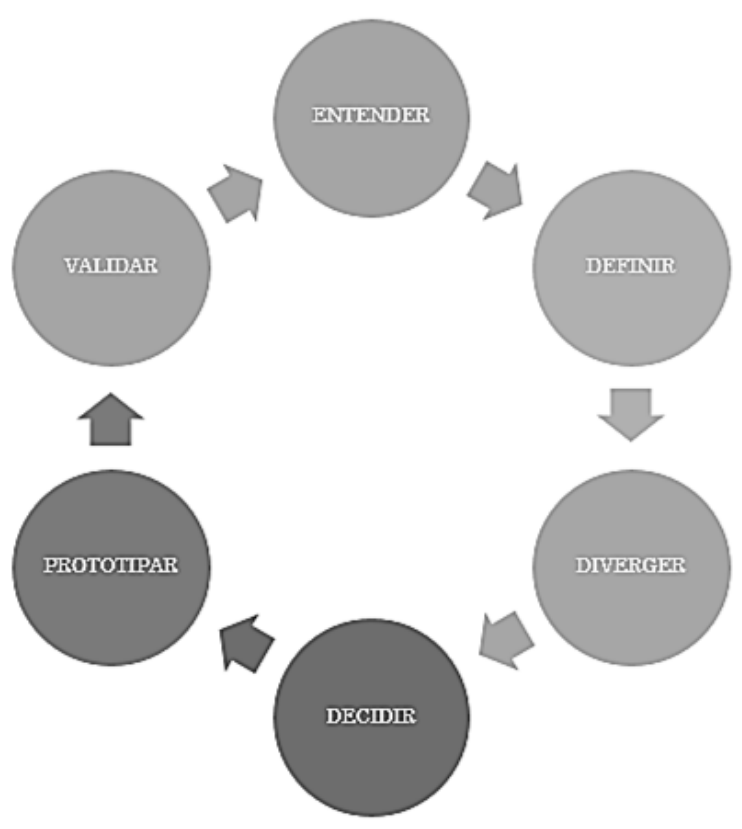

Figura 1. Fases metodológicas de diseño participativo. Fuente: Autores. Nota: Se muestran las 6 fases ejecutadas en el desarrollo de la estrategia de diseño participativo, que inició con la fase de entender y terminó validando los resultados de investigación.

La metodología de trabajo en la que se basó el desarrollo de las soluciones a los retos fue el Design Sprint. Este método combina ciertas actividades que, en conjunto, intentan responder a un problema o a una necesidad en el mercado, con una gama de ideas creativas y un prototipo probado (Moran \& Gonzalo, 2018).

Una vez hecha la caracterización de la población, se procedió con el diseño de la primera fase, que se muestra en la Figura 1 y corresponde a entender. En esta fase se buscó establecer unos retos municipales de innovación, con el fin de enmarcar todas las iniciativas, de acuerdo con las potencialidades del territorio.

De acuerdo con las actividades productivas que las mujeres desarrollan, se hizo un diagnóstico de las problemáticas o necesidades que presentaban. En esta fase fue de vital importancia que el facilitador tuviera la capacidad de identificar cuál de esas necesidades o problemáticas eran susceptibles de atender mediante el diseño participativo.

Para finalizar la primera fase de este proceso de innovación, se filtraron las problemáticas o necesidades, hasta llegar a definir tres de estas, que se debían enmarcar en los retos municipales de innovación. Definidas las tres problemáticas más relevantes, se procedió a conformar los grupos de trabajo, ya que una de las reglas para el desarrollo de la metodología es el trabajo en equipo.

La segunda fase, que corresponde a definir, buscó formularun reto de innovación a partir dela problemática o necesidad identificada previamente. Para plantear el reto, se debía responder a 4 interrogantes: qué, quién, por qué y si es un proyecto o un problema.

El qué permite definir el problema, el quién permite identificar el usuario o posibles clientes, el por qué define el beneficio que se genera al resolver el reto $y$, por último, si la iniciativa parte de un problema permitirá llegar a una solución que aún no se ha propuesto, pero si es un proyecto, quiere decir que ya se tiene una solución concebida y solo falta la etapa de ejecución; por tanto, no encajaba en la metodología planteada.

Una vez definido el reto de innovación se procedió a desarrollar la tercera etapa, divergir. En esta fase se buscó identificar tantas soluciones como fuese posible. En esta etapa las mujeres de ASOAMMES aportaron el conocimiento adquirido a través de la experiencia $\mathrm{y} / \mathrm{o}$ conocimientos heredados, mejor llamados "conocimientos ancestrales". Adicionalmente, fue necesario desarrollar una búsqueda de soluciones existentes, para lo cual se hizo uso de herramientas tecnológicas, como la búsqueda de patentes o productos en el mercado. Una estrategia para hacer más efectiva esta fase fue involucrar jóvenes rurales que se encuentran cursando grado $9 .^{\circ}, 10 .^{\circ}$ y $11 .^{\circ}$, con el objetivo de facilitar la recolección de información, por medio de consultas en bases de datos.

Una vez fueron identificadas las soluciones existentes, y con base en el conocimiento empírico o ancestral, se formularon 3 alternativas que daban solución a cada reto de innovación y que eran viables de desarrollar e implementar.

La fase de decidir correspondió al proceso de seleccionar la alternativa más viable a desarrollar. Para ello, fue necesario evaluar el grado de innovación, la complejidad y la factibilidad de implementación, de acuerdo con el contexto y los recursos con que se contaba para desarrollar las iniciativas.

Definida la solución a desarrollar, se buscó materializar las ideas mediante un proceso de prototipado básico. Este prototipo se elaboró con materiales básicos y de fácil manipulación. Algunos de estos fueron papel, cartón, plastilina, madera y objetos reutilizados. Con el prototipo se buscó representar formal y funcionalmente 
la solución propuesta, y de esta manera aprobar el diseño o realizarle ajustes.

Una vez definido el prototipo de la solución, se procedió a desarrollar la fase de validación. Para esta etapa se hizo una primera actividad de validación entre los grupos de trabajo y el facilitador. Posteriormente, cada grupo de trabajo debía salir a buscar posibles usuarios o clientes, para presentarles la idea y el prototipo de la manera más clara y rápida. Además, cada grupo debía llevar un registro de la impresión que causó la propuesta en el usuario y sus posibles sugerencias para mejorar la solución. Con las sugerencias aportadas por los grupos de trabajo y los usuarios externos, se hicieron los ajustes y mejoras tanto a la idea como al prototipo, para finalmente someter las iniciativas a una evaluación por parte de expertos.

Una vez definida y aprobada la propuesta de innovación, se procedió con la implementación. Para facilitar el desarrollo de cada una de las fases anteriormente expuestas, se realizó el diseño de formatos físicos, en los cuales se consignan los resultados de cada actividad. Lo anterior, con el fin de hacer seguimiento al proceso de innovación y de formular ajustes o estrategias para el óptimo desarrollo.

El procedimiento ya mencionado se desarrolló en cinco sesiones de trabajo. Estas fueron propuestas cada una de 4 horas, y con intervalos de ocho días entre estas. Ello con el fin de facilitar la participación de las mujeres, sin afectar sus labores cotidianas y productivas.

\section{Resultados}

En la fase de caracterización de la población, de las 40 mujeres que conforman la asociación, participaron 34, lo que corresponde al 85 \% de la población.

En la Tabla 1 se identifica que la edad promedio de estas mujeres es de 52 años, la mujer con mayor edad tiene 84 años y la mujer con menor edad tiene 30 años. En cuanto a la escolaridad, se encontró que el 5,8 \% de las mujeres no sabe leer ni escribir, el 70,6 \% tiene acceso a básica primaria, el 20,6 \% llegó a básica secundaria y el 2,9\% llegó a cursar estudios técnicos. Este último es el grado de escolaridad más alto que se encuentra en la comunidad (ver Figura 2).

\section{Tabla 1}

\section{Caracterización de la población}

\begin{tabular}{|c|c|c|c|c|c|c|c|c|c|c|c|}
\hline \multirow[t]{2}{*}{ Edad } & \multirow[b]{2}{*}{ Analfabeta } & \multirow[b]{2}{*}{ Primaria } & \multicolumn{2}{|l|}{ Escolaridad } & \multirow[b]{2}{*}{ Profesional } & \multicolumn{2}{|c|}{$\begin{array}{c}\text { Madre } \\
\text { cabeza } \\
\text { de } \\
\text { hogar }\end{array}$} & \multicolumn{2}{|c|}{$\begin{array}{c}\text { Procesos } \\
\text { de } \\
\text { diseño }\end{array}$} & \multirow{2}{*}{$\begin{array}{c}\begin{array}{c}\text { Cuidado } \\
\text { del hogar }\end{array} \\
\begin{array}{c}\text { Horas X } \\
\text { Día }\end{array} \\
\end{array}$} & \multirow{2}{*}{$\begin{array}{c}\begin{array}{c}\text { Actividad } \\
\text { productiva }\end{array} \\
\begin{array}{c}\text { Horas X } \\
\text { Día }\end{array} \\
\end{array}$} \\
\hline & & & Bachillerato & Técnico & & $\mathbf{S i}$ & No & Si & No & & \\
\hline 41 & 0 & 0 & 1 & 0 & 0 & 1 & 0 & 0 & 1 & 3 & 6 \\
\hline 50 & 0 & 0 & 1 & 0 & 0 & 1 & 0 & 0 & 1 & 4 & 4 \\
\hline 48 & 0 & 0 & 1 & 0 & 0 & 0 & 1 & 1 & 0 & 2 & 8 \\
\hline 52 & 0 & 1 & 0 & 0 & 0 & 1 & 0 & 0 & 1 & 3 & 8 \\
\hline 50 & 0 & 1 & 0 & 0 & 0 & 1 & 0 & 0 & 1 & 2 & 2 \\
\hline 54 & 0 & 1 & 0 & 0 & 0 & 1 & 0 & 0 & 1 & 4 & 8 \\
\hline 34 & 0 & 1 & 0 & 0 & 0 & 1 & 0 & 0 & 1 & 4 & 5 \\
\hline 50 & 0 & 1 & 0 & 0 & 0 & 1 & 0 & 0 & 1 & 12 & 3 \\
\hline 48 & 0 & 0 & 1 & 0 & 0 & 1 & 0 & 0 & 1 & 9 & 3 \\
\hline 66 & 0 & 1 & 0 & 0 & 0 & 1 & 0 & 0 & 1 & 8 & 5 \\
\hline 58 & 0 & 1 & 0 & 0 & 0 & 0 & 1 & 0 & 1 & 10 & 9 \\
\hline 59 & 0 & 1 & 0 & 0 & 0 & 1 & 0 & 0 & 1 & 24 & 8 \\
\hline 52 & 0 & 0 & 1 & 0 & 0 & 1 & 0 & 0 & 1 & 3 & 3 \\
\hline 30 & 0 & 0 & 1 & 0 & 0 & 1 & 0 & 0 & 1 & 10 & 10 \\
\hline 56 & 0 & 1 & 0 & 0 & 0 & 0 & 1 & 0 & 1 & 13 & 13 \\
\hline 74 & 0 & 1 & 0 & 0 & 0 & 1 & 0 & 0 & 1 & 12 & 12 \\
\hline 34 & 0 & 1 & 0 & 0 & 0 & 0 & 1 & 0 & 1 & 6 & 6 \\
\hline 61 & 0 & 1 & 0 & 0 & 0 & 1 & 0 & 0 & 1 & 10 & 2 \\
\hline 32 & 0 & 1 & 0 & 0 & 0 & 1 & 0 & 1 & 0 & 12 & 12 \\
\hline 52 & 0 & 1 & 0 & 0 & 0 & 0 & 1 & 0 & 1 & 12 & 2 \\
\hline 42 & 0 & 1 & 0 & 0 & 0 & 1 & 0 & 0 & 1 & 7 & 3 \\
\hline 72 & 0 & 1 & 0 & 0 & 0 & 1 & 0 & 0 & 1 & 4 & 6 \\
\hline 56 & 1 & 0 & 0 & 0 & 0 & 0 & 1 & 0 & 1 & 12 & 2 \\
\hline 31 & 0 & 1 & 0 & 0 & 0 & 1 & 0 & 0 & 1 & 2 & 10 \\
\hline
\end{tabular}




\begin{tabular}{lllllllllllll}
59 & 0 & 1 & 0 & 0 & 0 & 0 & 1 & 0 & 1 & 4 & 6 \\
44 & 0 & 1 & 0 & 0 & 0 & 0 & 1 & 0 & 1 & 8 & 2 \\
59 & 0 & 1 & 0 & 0 & 0 & 0 & 1 & 0 & 1 & 6 & 3 \\
52 & 0 & 1 & 0 & 0 & 0 & 1 & 0 & 0 & 1 & 2 & 1 \\
84 & 1 & 0 & 0 & 0 & 0 & 1 & 0 & 0 & 1 & 4 & 4 \\
62 & 0 & 1 & 0 & 0 & 0 & 1 & 0 & 0 & 1 & 12 & 2 \\
\hline
\end{tabular}

Fuente: Autores. Nota: Para efectos de la sistematización de la información, 0 es igual a no, y 1 es igual a sí. La edad, horas de dedicación al cuidado del hogar y actividades productivas se expresan tal como las encuestadas respondieron.

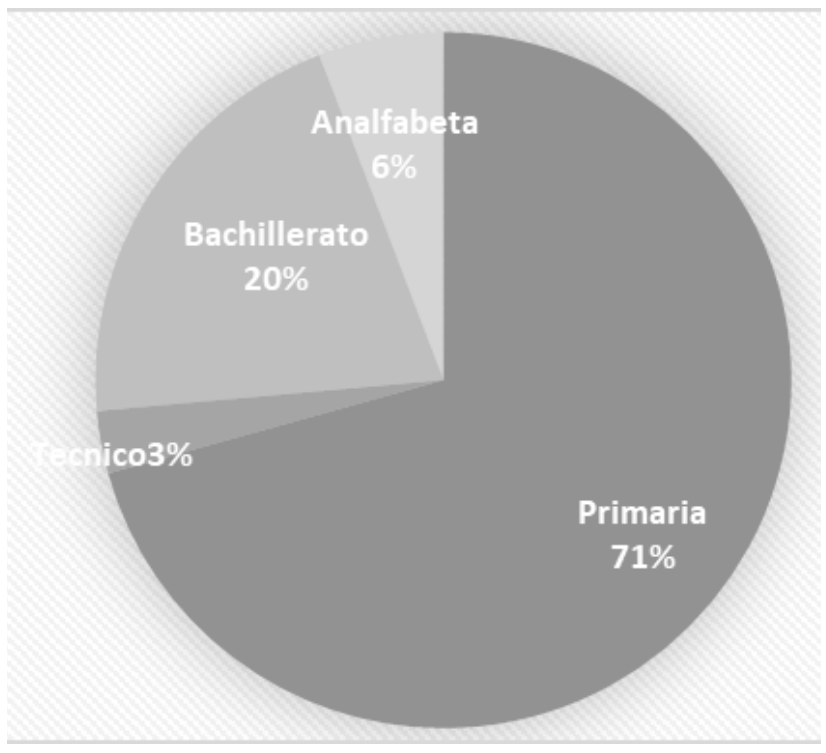

Figura 2. Grado de escolaridad de la población participante. Fuente: Autores. Nota: Datos obtenidos a través de la aplicación y análisis de encuesta.

De las 34 mujeres, el $70 \%$ son madre cabeza de hogar; y del total de la población, el número promedio de hijos es de 4. En la caracterización, se identificó que el $8,9 \%$ de las mujeres encuestadas ha oído los términos 'innovación' y 'diseño'. En cuanto al uso del tiempo, estas mujeres dedican en promedio 8 horas por día al cuidado del hogar y 5 horas al desarrollo de actividades productivas.

Las mujeres involucradas en el proceso de diseño participativo fueron nueve. Ellas propusieron voluntariamente desarrollar tres proyectos. El nivel de participación en estos talleres estuvo condicionado por disponibilidad de tiempo, disponibilidad de recursos para movilización al casco urbano, disponibilidad de transporte y voluntad e interés.

\section{Entender}

Como resultado de la fase entender, se propuso a partir de una caracterización de las actividades productivas, y luego plantear 3 retos municipales de innovación, para enfocar todas las iniciativas. Los retos municipales fueron construidos entre el facilitador, el secretario de fomento agropecuario y la comunidad participante en la primera sesión de trabajo. Esta etapa fue desarrollada entre el facilitador, la comunidad y los colaboradores, en una primera sesión de trabajo, que tuvo una duración de dos horas. Como insumos para su desarrollo, se elaboró una caracterización del municipio de Sotaquirá.

Retos municipales de innovación:

1. Desarrollar prácticas agrícolas sostenibles que permitan agregar valor a las cadenas productivas.

2. Fortalecer estrategias para desarrollar el turismo rural comunitario y sostenible que involucre el conocimiento ancestral de los habitantes del municipio de Sotaquirá.

3. Hacer uso eficiente de los recursos naturales en el desarrollo de las actividades económicas y de desarrollo en el municipio.

\section{Definir}

Luego de establecer los retos municipales de innovación $\mathrm{y}$ analizar las necesidades $\mathrm{y} / \mathrm{o}$ problemas expuestos por cada una de las mujeres que participaron en el proceso de investigación, se seleccionaron los temas a desarrollar, según el alcance, para dar solución a través del diseño. Algunas de las problemáticas expresadas por la comunidad inicialmente fueron falta de acceso a servicios públicos; carencia de vías de acceso; y dificultades en la comercialización de productos. Por ello, la labor tanto del facilitador como de los colaboradores se centró en enfocar a la comunidad en identificar necesidades o problemáticas meramente productivas, ya que el objetivo principal de la investigación fue incidir en el fortalecimiento de las actividades productivas. En esta fase fue de vital importancia establecer los grupos de trabajo de acuerdo con la actividad que desarrolla cada persona, y plantear los retos de innovación que respondieran a las problemáticas que cada participante expresó mediante una breve exposición verbal. Los criterios de selección de las problemáticas o necesidades de innovación estuvieron en función de la cobertura 
máxima de la población, de los alcances del proyecto de investigación y del beneficio esperado. Esta fase fue desarrollada durante el espacio de trabajo planeado y no requirió dejar actividades extra a la comunidad.

Retos de innovación:

1. ¿Cómo optimizar el proceso de recolección de mora para garantizar calidad e inocuidad del fruto y seguridad al operario, a través de un equipo o dispositivo?

Para la construcción de este reto se identificaron las siguientes problemáticas:

Laceraciones en las manos de las mujeres, que son causadas por el contacto de la piel con las espinas del árbol de la mora.

Por exigencia del mercado, la mora no debe llevar pedúnculo; por tanto, al realizar el proceso de cosecha de manera manual, se requiere desarrollar un posproceso para retirar dicho pedúnculo.

Por manejo de buenas prácticas agrícolas, la comunidad manifestó que se debía garantizar la innocuidad del producto, y al agarrar el fruto directamente con la mano, se podría contaminar con sudor o incluso tener contacto con las heridas causadas por las espinas.

Al agarrar los frutos directamente con la mano, se podía maltratar la mora, por exceso de presión, al intentar desprenderla del árbol.

El método de cosecha tradicional se hace depositando el fruto directamente en la canastilla. Esta es difícil de transportar dentro del cultivo, por el volumen y por el peso.

2. ¿Cómo controlar el daño en los cultivos de hortaliza causado por el caracol, de una manera eficiente, que no altere el $\mathrm{pH}$ de la tierra, ni químicamente contamine las plantas, para aumentar la productividad y calidad en los productos agrícolas?

Para formular este reto de innovación se tuvieron en cuenta las siguientes variables:

Todas las asociadas poseen cultivos, ya sea de frutas, verduras o tubérculos. Estos se han visto afectados por presencia de caracol, lo que disminuye el volumen de producción de las parcelas.

La especie de caracol ha desarrollado cierta inmunidad a insecticidas comerciales; además, ASOAMMES busca producir alimentos orgánicos libres de químicos, lo que hace inviable la utilización de insecticidas.

3. ¿Cómo promocionar y comercializar los productos orgánicos de ASOAMMES para obtener un mayor beneficio económico y llegar a atender clientes como autoservicios, almacenes de cadena y supermercados?

Para formular este reto de innovación, se tuvieron en cuenta las siguientes variables:

El empacado de los productos vendidos por unidad se hace en bolsas comerciales, y estas no brindan información del producto ni aportan un factor diferenciador en el mercado.

La venta de productos al por mayor se hace en canastillas en las que no se garantiza la conservación del producto.

El desarrollo de una línea de empaques se hace necesaria para incursionar en mercados como las superficies de cadena.

Se llegó a formular estos retos de innovación mediante el acompañamiento del facilitador y los colaboradores a cada uno de los grupos de trabajo. La comunidad aportó la información a través de una socialización de las problemáticas o necesidades, y el facilitador contribuyó con la redacción de los retos.

\section{Divergir}

Una vez planteados los retos de innovación, se procedió a explorar posibles soluciones. La primera instancia fue identificar soluciones basadas en conocimiento ancestral o empírico, seguido por una búsqueda de soluciones existentes.

Esta fase se desarrolló dentro del espacio planeado. Adicionalmente, fue necesario que la comunidad hiciera una búsqueda de ideas, mediante la indagación con familiares, amigos, vecinos, establecimientos comerciales y expertos, acerca de posibles soluciones al reto planteado. Una tarea indispensable del facilitador fue hacer búsquedas exhaustivas de posibles soluciones a los retos de innovación, y de esta manera poder validar la información de la comunidad.

Como resultado de esta fase, se obtuvieron posibles ideas, como la implementación de una punta de tijera que podría ser una solución para cortar el pedúnculo de la mora. Se encontraron dispositivos cosechadores de gran escala, y también cosechadores de accionamiento manual para la recolección de frutos de mayor tamaño, 
como la naranja. En el mercado, sin embargo, no se encontró un dispositivo cosechador de mora.

Dentro de las posibles soluciones identificadas como respuesta al segundo reto de innovación, se encontró la utilización de insecticidas en el perímetro de los cultivos de trampas con sal o cerveza, y aun dispositivos electrónicos. En el proceso de búsqueda de alternativas para controlar el caracol, se halló que la helicicultura o cría de caracoles es una de las iniciativas de emprendimiento en la actualidad, y se propuso que a corto o mediano plazo la asociación podría incursionar en la helicicultura como una actividad productiva alternativa. Sin embargo, para dar solución al reto planteado, se propuso explorar técnicas conocidas en las que se involucre el diseño de trampas con materiales caseros.

Para dar solución al tercer reto de innovación en la búsqueda de soluciones existentes, se encontró una línea de empaque comercial que podría ser implementada para el empacado de la mora; no obstante, al analizar este referente, se determinó que carece de ventilación; por tanto, provoca una sudoración del producto empacado. Con seguridad, hacen un solo uso del empaque, y por consiguiente generarían residuos contaminantes. Las presentaciones que vienen dadas son muy pequeñas para empacar productos como frutas o verduras.

\section{Decidir}

En esta fase se seleccionaron las ideas que mejor respondían a los retos de innovación, para su posterior desarrollo y materialización en la fase de prototipado. Esta fase fue desarrollada entre el facilitador y los grupos de trabajo, junto con los colaboradores dentro del espacio de trabajo planteado, y basados en la información recolectada en la fase anterior. Las ideas seleccionadas para desarrollar fueron las siguientes:

Reto 1: Desarrollo de un dispositivo cosechador que permita hacer el corte del pedúnculo, con el fin de ofrecer frutos que cumplan con las buenas prácticas agrícolas y sean susceptibles de venta en mercados especializados, así como garantizar la seguridad del operario, para, por ejemplo, evitarle laceraciones en las manos.

Reto 2: Diseño de trampas para el control de caracol, a partir de materiales reutilizados que se encuentren en los hogares.

Reto 3: Diseño de una línea de empaques de múltiples usos que permitan almacenar frutas y verduras sin presentar sudoración.

\section{Prototipar}

Esta fase fue desarrollada en su etapa inicial, mediante una descripción en la que los participantes planteaban el objetivo de desarrollar el prototipo, los materiales necesarios y los resultados esperados. Para el desarrollo de esta fase fue necesaria la presentación de los materiales disponibles para el prototipado y su forma de manipulación. Algunos de estos fueron plastilina, cartón, láminas de acetato y elementos del hogar, que fueron suministrados por el facilitador.

Seguido de esto se procedió con la materialización de las ideas, para lo que se desarrolló el prototipo en una fase inicial dentro del espacio de trabajo, como se muestra en la Figura 3. La culminación del prototipo estuvo a cargo de los grupos de trabajo propuestos fuera de los talleres planeados.

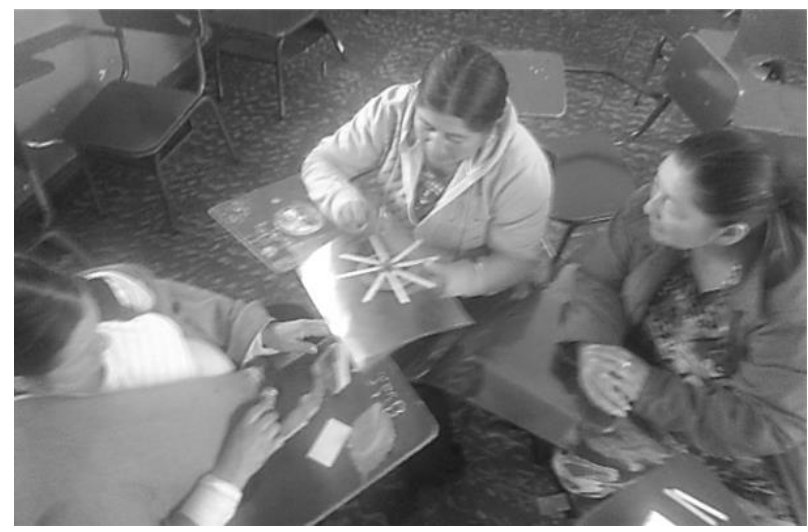

Figura 3. Taller de prototipado. Fuente: Autores. Nota: En la fase de prototipado se desarrolló una actividad de materialización de ideas, por medio de materiales básicos y de fácil manejo.

Los prototipos desarrollados por la comunidad debían representar formal y funcionalmente las propuestas seleccionadas para dar respuesta a los retos (Figuras 4, 5 y 6$)$.

El prototipo del dispositivo cosechador de mora planteaba el uso de unas cuchillas en forma de tijera que permitieran cortar el pedúnculo, para que el fruto bajara por un ducto al sistema de almacenamiento.

El prototipo de las trampas para caracoles consistió en la representación de diferentes elementos. En estos se ponía alimento del gusto de esta especie, para atraerla, y con ello poder capturar al caracol de manera mecánica. Como respuesta al tercer reto de innovación, se propuso la línea de empaques que se muestra en la Figura 6. Se 
propusieron presentaciones de 1, 3 y $5 \mathrm{~kg}$. Los empaques propuestos eran para varios usos, con el objetivo de reducir desechos. Además, se propuso una identidad gráfica de los empaques.

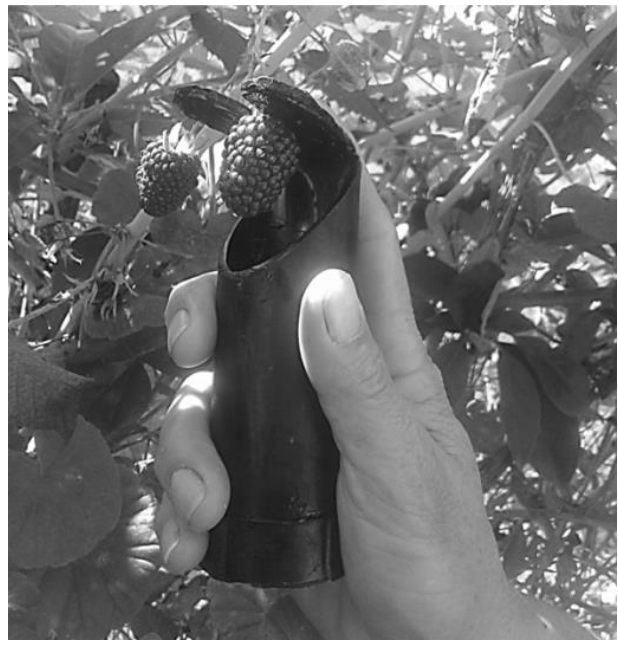

Figura 4. Prototipo del dispositivo cosechador de mora. Fuente: Autores. Nota: Elaborado con materiales reutilizados; se propuso la forma de uso.

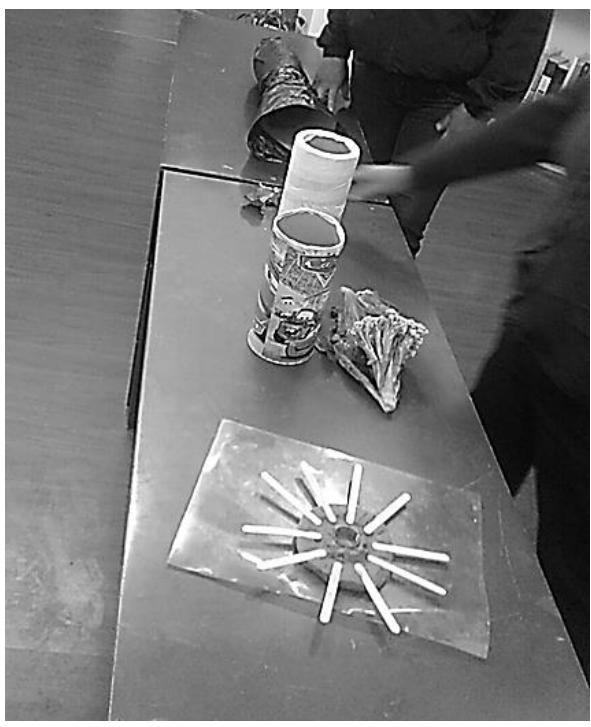

Figura 5. Prototipos de trampas para caracoles. Fuente: Autores. Nota: Prototipos desarrollados para dar solución a la presencia de caracol en los cultivos de hortaliza.

\section{Validar}

En esta fase de la metodología de trabajo se expusieron los prototipos a personas externas al proceso de innovación, con el fin de mejorar las propuestas desarrolladas. Una vez definidos los prototipos, cada iniciativa de innovación fue sometida a evaluación por parte de expertos, como la directora de externalización de procesos de conocimiento, de INNVENTTO S. A. S., spin off que hace parte del ecosistema de innovación de Medellín; y el director del Instituto de Gestión, Innovación y Aceleración de Negocios (GINNOA), ya que este instituto busca la financiación de iniciativas de innovación. El formato de evaluación fue el siguiente:

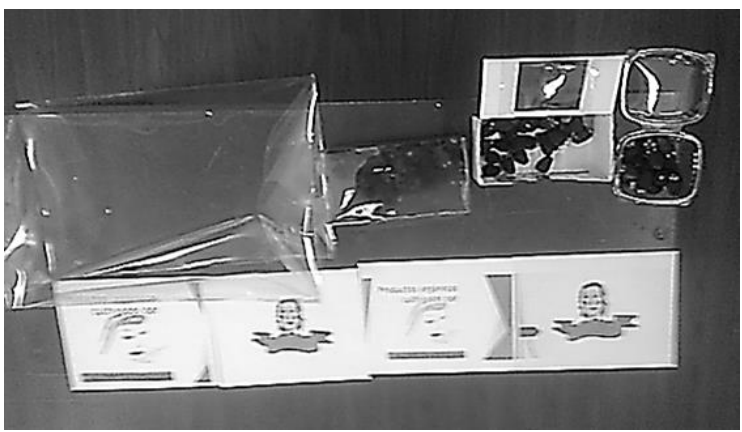

Figura 6. Prototipo de empaques y propuesta de identidad gráfica. Fuente: Autores.

\section{Tabla 2}

Variables por evaluar en las iniciativas de innovación

\begin{tabular}{ccl}
\hline $\begin{array}{c}\text { Criterio de } \\
\text { Evaluación }\end{array}$ & $\begin{array}{c}\text { Peso del } \\
\text { criterio en } \\
\text { la } \\
\text { evaluación } \\
\text { final }\end{array}$ & $\begin{array}{c}\text { Descripción del } \\
\text { criterio }\end{array}$ \\
\hline Usuario & $12 \%$ & $\begin{array}{l}\text { Claridad en el } \\
\text { usuario objetivo y el } \\
\text { potencial de } \\
\text { aceptación de la } \\
\text { solución propuesta. }\end{array}$ \\
Reto & $20 \%$ & $\begin{array}{l}\text { Relación del reto } \\
\text { propuesto en la } \\
\text { primera fase con la } \\
\text { solución generada. }\end{array}$ \\
Propuesta de & $14 \%$ & $\begin{array}{l}\text { Factor diferenciador } \\
\text { de la solución. }\end{array}$ \\
valor & $12 \%$ & $\begin{array}{l}\text { Posibilidad de que la } \\
\text { solución se sostenga } \\
\text { a largo plazo, una } \\
\text { vez termine la }\end{array}$ \\
intervención. \\
Viabilidad
\end{tabular}

Fuente: Autores. 
Como resultado de este proceso de evaluación por parte de expertos, se obtuvo la financiación para desarrollar e implementar el dispositivo cosechador de mora que se muestra en la Figura 8.

Una vez culminados los prototipos y evaluadas las iniciativas de innovación, se procedió con el diseño e implementación del dispositivo cosechador de mora. Las otras dos iniciativas quedaron en fase de prototipos, por falta de recursos para su ejecución e implementación. Con todo, se espera desarrollarlas en la medida que haya disponibilidad de recursos económicos.

El desarrollo del dispositivo cosechador de mora estuvo a cargo del facilitador, quien fue el encargado de la elaboración de los planos técnicos requeridos para el proceso de manufactura, la selección de materiales y elección de procesos de manufactura. Esta fase fue desarrollada con la aprobación de la comunidad, para garantizar el prototipado funcional de la propuesta.

Una vez elaborados los planos técnicos, definidos los materiales, procesos y recibida la aprobación de la comunidad, se encomendó el proceso de manufactura y ensamble a una empresa del sector metalmecánico de la región.

\section{Dispositivo cosechador de mora}

Este dispositivo fue propuesto por la comunidad ASOAMMES y constaba de:

- Un arnés que permite transportar el recipiente de almacenamiento por parte del operario, que está elaborado en reata y hebillas para facilitar su uso.

- Recipiente de almacenamiento, esto es, un elemento comercial seleccionado que tradicionalmente es utilizado en la recolección de café. Se trata de un ducto flexible que lleva la mora del dispositivo de corte al recipiente de almacenamiento. Este ducto es apto para el contacto con alimentos. Su flexibilidad le permite al operario manipular fácilmente el dispositivo.

- Dispositivo de corte, que tiene un mecanismo de tijera que permite cortar el pedúnculo del fruto. Este es accionado de manera manual, por medio de una palanca que se encuentra en la empuñadura del dispositivo. Esta sección del prototipo fue elaborada en acero inoxidable referencia 304, el cual es apto para contacto con alimentos. Los procesos de manufactura fueron cote por arranque de viruta y soldadura.

En la Figura 7 se puede observar el sistema de corte del dispositivo cosechador de mora, compuesto por una mordaza fija y una móvil que se acciona por medio de un sistema de palanca y resorte. El cuerpo del dispositivo está elaborado en tubo de acero inoxidable, por el cual cae el fruto y es conducido al recipiente de almacenamiento.

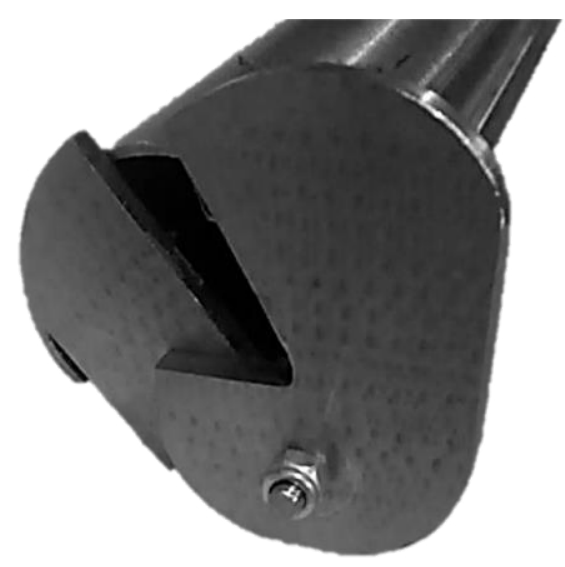

Figura 7. Sistema de corte del dispositivo cosechador de mora, con mordazas fija y móvil, soportadas sobre un tubo de acero inoxidable. Fuente: Autores.

La implementación del dispositivo cosechador de mora se hizo en la finca de una de las beneficiarias del proyecto. Mediante esta actividad, se logró evidenciar la seguridad que ofrece al operario al evitar las laceraciones en las manos producidas por las espinas del árbol. También se comprobó el corte del pedúnculo, el cual no es deseado a la hora de comercializar el fruto. El aspecto físico de la mora es mejor al ser recolectada con este dispositivo, ya que no recibe presión por parte del operario al intentar arrancarla del árbol. Una desventaja en la recolección de mora que se evidenció con la implementación de este dispositivo fue la reducción en la productividad, ya que la medición arrojó que el operario recolecta con el dispositivo un $30 \%$ menos de lo que recolecta de manera manual. La medición se hizo en kilogramos por hora, de modo que ello se constituye como un aspecto para la mejora del cosechador de mora.

Ya que el desarrollo del dispositivo cosechador de mora fue un proceso que llegó a presentar un prototipo funcional, gracias a la financiación de materiales y procesos de manufactura, el grupo de trabajo que desarrolló la iniciativa fue invitado a participar en el primer workshop de innovación organizado por el centro de convenciones de Tunja. En este espacio, estas mujeres y el facilitador de la metodología de innovación dieron a conocer los resultados del proceso. 


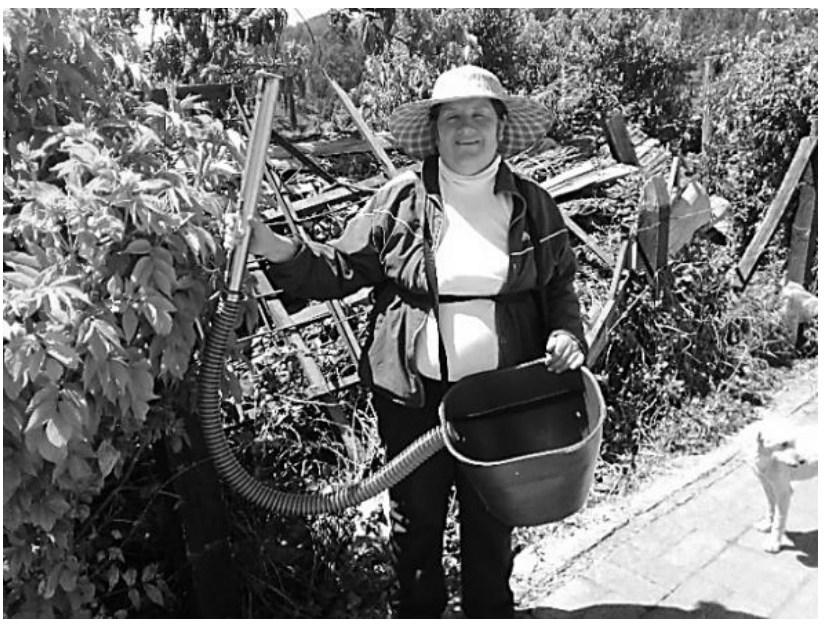

Figura 8. Implementación del dispositivo cosechador de mora en el municipio de Sotaquirá por la líder de ASOAMMES. Fuente: Autores.

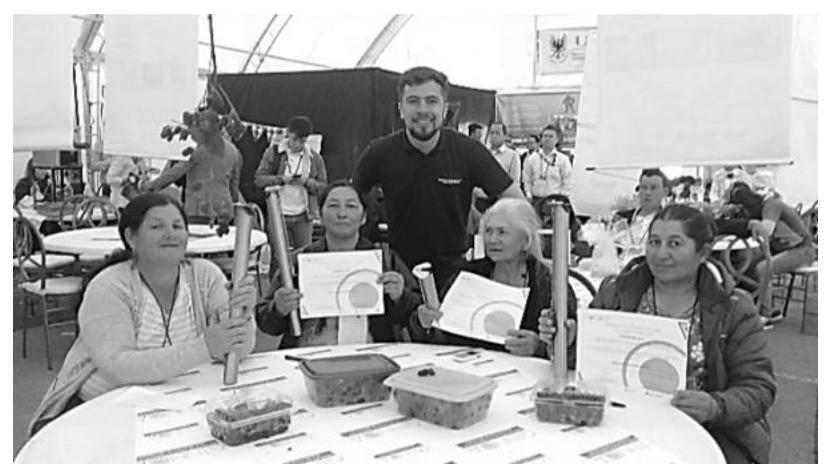

Figura 9. Grupo de trabajo que desarrolló el dispositivo cosechador de mora en el primer workshop de innovación, en el centro de convenciones de Tunja (22 de noviembre de 2018). Fuente: Autores.

Luego de 45 días de finalizados los talleres de diseño participativo, las mujeres que hicieron parte del proceso desarrollaron una línea de empaque reutilizable. Según dieron a conocer, esta idea surgió a raíz de la necesidad de tener un empaque para la papa que están produciendo. La línea de empaques que desarrollaron (ver Figura 10) consta de 3 piezas elaboradas a partir de materiales que tienen en su entorno, como fique, cabuya e hilo. Cada una de las piezas está elaborada en un material diferente y en diferentes formas de tejido. Además, están hechas para empacar $5 \mathrm{~kg}$ de papa. Cabe destacar que estos empaques fueron pensados para varios usos, lo que da a conocer que quienes los hicieron estaban conscientes de conceptos como la sostenibilidad.

De acuerdo con lo expresado por quien lidera la asociación ASOAMMES, a raíz de la necesidad de ofrecer sus productos con un factor diferenciador en el mercado campesino local, tomaron la decisión de poner en práctica los conocimientos adquiridos en los talleres de diseño participativo, quizá no en su totalidad ni en la secuencia desarrollada en los talleres dirigidos, pero sí aplicando conceptos clave. Algunos de estos conceptos fueron la identificación de la problemática, el análisis de soluciones existentes y el factor diferenciador en el producto.

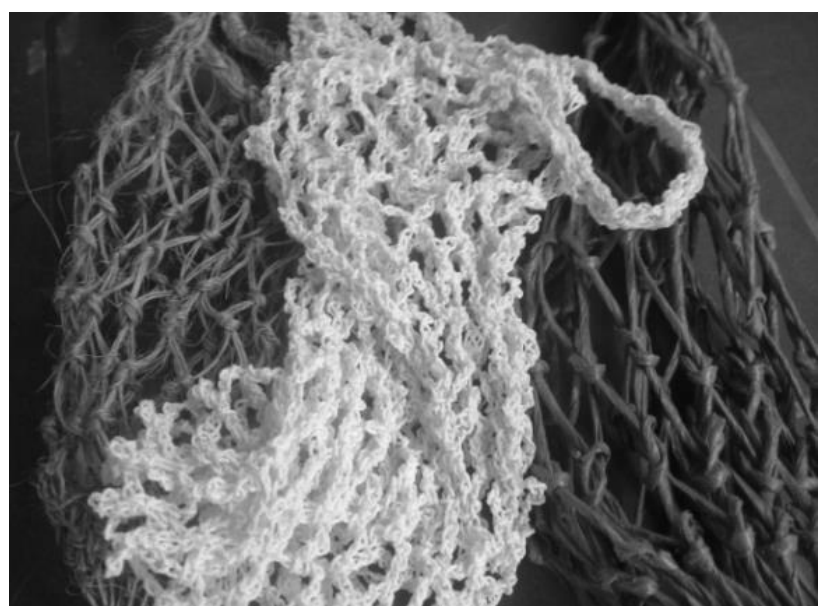

Figura 10. Línea de empaques para papa. Fuente: Autores. Nota: Se muestran tres tipos de empaques para papa, diseñados y elaborados por las mujeres que participaron en el proceso de investigación e innovación.

\section{Conclusiones}

Además de cumplir el objetivo general propuesto para esta investigación, es importante resaltar el aporte que se ha realizado con este caso de estudio: la transferencia de conceptos y herramientas de innovación a la comunidad femenina rural de Sotaquirá ASOAMMES. Estos conceptos y herramientas han sido apropiados para dar solución a los retos productivos presentes y futuros, lo que genera un empoderamiento, al fortalecer las capacidades de las personas y resaltar sus logros. Esta transferencia y apropiación de conceptos y herramientas se evidencia en las tres iniciativas de innovación que propuso la comunidad, con la implementación del dispositivo cosechador y con la propuesta de una línea de empaques desarrollada de manera autónoma por la comunidad.

Esta investigación cobra relevancia, ya que en los ámbitos local, regional, nacional y latinoamericano existen comunidades femeninas que aportan en gran medida al desarrollo de los territorios, aun cuando se encuentran con mayor riesgo de vulnerabilidad. Así, mediante un enfoque participativo del diseño y metodologías ágiles 
de innovación, se puede llegar a incidir tanto en las condiciones de vida como en el desarrollo de actividades productivas con mayor valor agregado.

Para la disciplina del diseño, el trabajo con comunidades rurales es un campo sin profundizar, pero a la vez una gran posibilidad para el desenvolvimiento profesional y para el desarrollo de proyectos de investigación e innovación con alto impacto, tanto productivo como social.

\section{Recomendaciones}

Como recomendación a nuevas iniciativas relacionadas, una vez obtenidos los resultados de la investigación, se debe mantener un acompañamiento y seguimiento a la comunidad, tanto para apoyarlos en nuevos procesos como para evaluar su continuidad.

Un sesgo importante de resaltar en este proceso de investigación es la participación de las personas en los talleres de diseño participativo, ya que en este caso se trabajó con un grupo reducido de personas. Esto permitió la facilidad en el desarrollo de las actividades, tanto para el facilitador como para los participantes y colaboradores. Posiblemente, y en la medida en que la participación de la comunidad es más significativa, se tendrán que ajustar las dinámicas para el desarrollo de estrategias como la presentada en este caso de estudio.

Por tanto, uno de los grandes retos es incrementar el nivel de participación de las personas en procesos de investigación e innovación como el desarrollado. Para esto, se deben considerar variables como duración de los talleres, frecuencia de los talleres, logística para la movilización de las personas, lugar de desarrollo de los talleres, recursos técnicos y estímulos para la implementación de las iniciativas. Por esto, se hace necesario involucrar a entidades públicas y/o privadas que estén interesadas en contribuir con este tipo de proyectos.

La sugerencia para futuras investigaciones o proyectos relacionados es la presentación de herramientas y equipos para el prototipado de las iniciativas de innovación, lo que permitirá el desarrollo más profundo de prototipos entre la comunidad y el facilitador. La comunidad podrá conocer nuevos procesos que la llene de ideas y motivación.

\section{Agradecimientos}

Al Instituto de Gestión, Innovación y Aceleración de Negocios (GINNOA), el Instituto de Investigación e
Innovación para la Competitividad Regional (CIMADI) y el grupo de investigación DITMAV, de la UPTC, sede Duitama, quienes apoyaron este proceso de investigación aportando materiales para el desarrollo de los talleres participativos; formación como gestores de innovación y financiación de la implementación del dispositivo cosechador de mora, mediante los recursos obtenidos por el convenio interadministrativo N. ${ }^{\circ} 839$, entre el Ministerio de Educación Nacional y la Universidad Pedagógica y Tecnológica de Colombia. Por último, un agradecimiento especial a la Asociación Agropecuaria de Mujeres Emprendedoras del Municipio de Sotaquirá, por su interés y compromiso en el desarrollo de la investigación.

\section{Referencias}

Botello-Peñalosa, H. A., \& Guerrero-Rincon, I. (2017). Condiciones para el empoderamiento de la mujer rural en Colombia. Entramado, 13(1), 62-70.

Castillo, M. (2011). Indicadores de Empleo de los Objetivos de Desarollo del Milenio dentro del marco de la medicion del trabajo decente. San Jose, Costa Rica: Organizacion Internacional del Trabajo.

Colmenares, A. M. (2012). Investigación-acción participativa: una metodologia integrara del conocimiento y la accion. Voces y Silencios: Revista Latinoamericana de Educacion, 3(1), 102-115.

CORPOICA. (2015). Diagnóstico de la Ciencia, Tecnología e Innovación en el Sector Agropecuario (Misión para la Transformación del Campo). Bogota D.C.: Corporación Colombiana de Investigación Agropecuaria.

Eizagirre, M., \& Zabala, N. (2019). Investigación-acción participativa (IAP) Diccionario de Accion Humanitaria y Cooperacion al Desarrollo. Recuperado de http:// www.dicc.hegoa.ehu.es/listar/mostrar/132

Escuela de diseño social. (02 de 11 de 2018). Mapa de oportunidad social. Recuperado de http://www. escueladisenosocial.org/la-escuela-de-disenosocial/

Freire, P. (1970). Pedagogía del oprimido. Montevideo: Tierra Nueva.

Romera, C., Suarez , F., \& Hernandez, J. A. (2017). Tecnología y diseño para el desarrollo rural: propuesta metodológica y prueba de concepto en dos municipios colombianos. Revista de Estudios sobre Despoblación y Desarrollo Rural, (23) 27-57.

Kawulich, B. B. (2006). La observación participante como método de recolección de datos. Forum Qualitative Sozialforschung / Forum: Qualitative Social Research [On-line Journal]. Recuperado de http://nbn-resolving. de/urn:nbn:de:0114-fqs0502430.

Manzini, E. (2015). Design, When Everybody Designs: An Introduction to Design for Social Innovation. 
Cambridge.

Moran, Y., \& Gonzalo, E. (2018). UX A través del Sprint de diseño. Universitat de Valencia. Recuperado de https://www.uv.es/capgeminiuv/documents/UX_ Sprint.pdf

Muñoz, A. J., Peña, Y. Y., \& Vargas, A. (2017). El rol de la asociatividad en la denominación de origen. $I+D$ Revista de Investigaciones,9(1) 27-37. https://doi. org/10.33304/revinv.v09n1-2017003

Naciones Unidas en Colombia. (2018). Objetivos de desarrollo sostenible para Colombia. Recuperado de https://nacionesunidas.org.co/ods/objetivo-5-lograrla-igualdad-entre-los-generos-y-empoderar-a-todaslas-mujeres-y-las-ninas/

Olarte, M. Y. (2015). Análisis del perfil de las cadenas productivas del café, la pitahaya, el limon Tahiti, desde un enfoque de internacionalizacion, innovacion y responsabilidad social empresarial en Bucaramanga. I $+D$ Revista de Investigaciones, 6(2), 76-99. https://doi. org/10.33304/revinv.v06n2-2015006

Nicolás, J. O. (2016). Diseñando el cambio. La innovación social y sus retos. Economía creativa, (6), 9-34.

Paz,A.I.,Sanchez,I.J.,\&Sanchez,J.A.(2018). Responsabilidad social factor integrador en Universidades Publicas. I+ D Revista de Investigaciones, 12(2) 37-46. https://doi. org/10.33304/revinv.v12n2-2018004 